\title{
Management of Traumatic Bile Leakage
}

\author{
Naoki Hashimoto \\ Department of Emergency Medicine, Sanda City Hospital, Keyakidai Sanda City, Hyogo, Japan \\ Email: gojigen000@gmail.com
}

How to cite this paper: Hashimoto, N. (2019) Management of Traumatic Bile Leakage. Open Access Library Journal, 6: e5556.

https://doi.org/10.4236/oalib.1105556

Received: June 20, 2019

Accepted: July 15, 2019

Published: July 18, 2019

Copyright $\odot 2019$ by author(s) and Open Access Library Inc.

This work is licensed under the Creative Commons Attribution International License (CC BY 4.0).

http://creativecommons.org/licenses/by/4.0/

\begin{abstract}
Major bile leak after blunt liver trauma (BLT) is rare but challenging. Data on endoscopic management of traumatic bile leaks are scare. We conducted a retrospective review to evaluate the role of Endoscopic retrograde cholangiography (ERCP) in the management of bile leaks following blunt abdominal trauma. In addition to a high grade injury, centrally-located liver injuries and initial TAE are also a significant risk factor for major bile duct injury. When patients after BLT are suffering from high fever, persistent abdominal pain or fullness, gross jaundice, we perform abdominal CT to detect intraabdominal fluid collection. If we aspirated bile from intraabdominal fluid, we made a diagnosis of bile duct injury highly possible and ERCP therapy was indicated due to high possibility of major bile leak and to provide early intervention.
\end{abstract}

\section{Subject Areas}

Emergency \& Critical Care, Gastroenterology \& Hepatology

\section{Keywords}

Bile Leak, Blunt Liver Injury, ERCP, TAE

\section{Introduction}

Blunt liver trauma (BLT) can be serious and lethal. Bile leaks can result from penetrating injury, such as gunshot or knife wounds or from blunt trauma such as motor vehicle accidents [1]. Following blunt abdominal trauma, there is significant damage to the biliary tract leading to an intrahepatic biloma, intraperitoneal leakage of bile, hemobilia and bilhemia [2]. The incidence of bile leaks following hepatobiliary trauma ranges from $0.5 \%-2.1 \%$ depending on the criteria and methods used to diagnose the bile leak [3].

Unlike iatrogenic bile duct injuries, bile duct injuries with bile leak after BLT are much more complex and subtle. The presentations are often nonspecific at 
first and an early precise diagnose is not easy.

Surgical procedures are associated with high complication rates due to the severity of accompanying inflammation, induration of the surrounding tissue, and presence of dense adhesions [4].

Endoscopic retrograde cholangiography (ERCP) has been found to be a useful tool for the diagnosis and treatment of post-traumatic bile leaks, but data on outcome after therapeutic ERCP for traumatic bile leaks is limited [2].

Endoscopic techniques used to treat bile leaks include biliary sphincterotomy alone, biliary stenting with or without sphincterotomy, and naso-biliary drainage with or without sphincterotomy. This allows internal drainage of bile and reduction in intrabiliary pressure, allowing the bile duct injury to seal off.

We conducted a retrospective review to evaluate the role of ERCP in the management of bile leaks following blunt abdominal trauma.

\section{Bile Leak Evaluation}

Biliary ductal injuries may not be seen on initial imaging or laparotomy and can therefore have an insidious presentation. The biliary injuries that result from hepatic trauma may be simple bile leaks into the lacerated liver, peritoneal cavity or pleural cavity. Bile leaks usually present several days after the initial trauma, with the patient complaining of abdominal pain, increasing abdominal distension, and ascites. In the setting of penetrating abdominal trauma such as gunshot, presentation of bile leak can be delayed up to two weeks as was reported previously [5].

When patients after BLT are suffering from high fever, persistent abdominal pain or fullness, gross jaundice, we performed abdominal CT to detect intraabdominal fluid collection. If abnormal intra-abdominal fluid collection was detected in abdominal CT, we aspirate intraabdominal fluid by echo. If we aspirated bile from intraabdominal fluid, we made a diagnosis of bile duct injury highly possible and ERCP therapy was indicated due to high possibility of major bile leak and to provide early intervention.

\section{Injury Location}

The biliary injury can be intrahepatic, extrahepatic or both. In previous studies, the right intrahepatic duct was the predominant series of bile leaks following abdominal trauma [6]. In a series by Sharma et al. [7], eight of ten patients had a bile leak from right hepatic duct after BLT. In a series by Singh et al. [2], bile leak was found to arise from right ducts in $70 \%$ and left ducts in $30 \%$ of cases. In a series by Bridges et al. [8], bile leaks were located in the right ductal system in nine (90\%) patients and in common hepatic duct in one patient.

Whether or not the location of the bile leak can predict treatment outcome is still unclear and cannot be elicited from M P Spinn's data [9]. But I think injury location is another significant factor for major bile leak. Yuan et al. [10] say that centrally located liver injuries (64.3\%) were more likely to develop major bile 
leak than peripherally located one $(0 \%)$. We considered injury location as a reasonable and relevant risk factor. Because the intrahepatic bile ducts distribute in a confluent manner and the main bile ducts are located more centrally. Main bile duct is more likely to be injuried if the blunt liver trauma involves the central parts. When the main bile duct is injuried, it is more difficult to recover than the peripheral located small bile ducts, and major bile leak is therefore more likely to happen.

\section{Management for Bile Leak}

The conventional treatment for post-traumatic bile leaks has been surgery. Surgery is often difficult to perform, however, due to adhesions or inflammation in and around the damaged liver parenchyma, or the patient may be poor surgical risk. Surgical treatment can often result in complications like stricture formation and cholangitis. The presence of other associated injuries involving the head, chest or extremities may further complicate the management of such patients. ERCP has been used as a diagnostic as well as therapeutic modality for biliary injuries in patients with liver trauma in adults as well as children. ERCP helps in assessing the site of both extrahepatic and intrahepatic bile leaks and provides information regarding hemobilia and pancreatic duct injury.

When biliary leak was treated by ERCP, a7 or 10 French plastic stent was inserted with or without endoscopic sphincterotomy (ES).

The ERCP was performed with a therapeutic duodenoscope (TJF 140R, Olympus opticals LTD, Tokyo Japan). While performing biliary stenting, no attempt was made to place the stent across the injuried duct. The stent was left in the distal ductal system to aid bile drainage. Post ERCP follow up was monitored by clinical assessment and by monitoring of bile output from the drains. Resolution of bile leak was documented on repeat ERCP which was anywhere from 4 to 16 weeks after initial ERCP. Sharma et al. [7] documented resolution of bile leak after 8.5 days of endoscopic interventions. In a series by Singh et al. [2], bile leaks closed in 15.76 days in patients who had undergone sphincterotomy, and in 12.14 days in patients without sphincterotomy. Lubezky et al. [11] reported resolution of bile leaks in 6 - 7 days after ERCP. Sugiyama et al. [12] reported healing of bile leaks after 1 - 7 days of ERCP. Bajaj et al. [13] reported time to resolution of bile leak after ERCP as 7.4 days.

Recovery was defined as the cessation of leakage or absence of radiological evidence of a biliary leak. Drains were removed once recovery had been achieved. The ideal length of time for stenting is not known. Stent removed was planned after insertion in previously published reports from 3 to 8 weeks [2]. M P Spinn et al. [5] removed the stents after an interval of 4 - 16 weeks. We routinely perform biliary sphinctrotomy and endobiliary stent placement for the management of a leak. In major leaks it is unlikely that sphincterotomy alone can seal the leak. I think ERCP with sphincterotomy and endobiliary stent placement are good effect for management of patients with extensive traumatic 
hepatic injury with intrahepatic bile leaks. ERCP should be considered as a fast line therapy for traumatic bile leaks.

\section{Are Initial TAE Also Significant Risk Factor for Major Bile Duct Injury?}

The initial management method for blunt liver trauma is also relevant for major bile leak in Yuan's study [10]. Patients who received TAE for initially have significant higher incidence of major bile leak in Yuan's study than patients who received surgery or non-operative management (NOM). A similar result had been reported. Wahl [14] reported the incidence of bile leak in patients who received TAE is $41.2 \%$; whereas it is only $19.2 \%$ for those who received operation; and $1.5 \%$ for those who were only observed [15]. Since major hepatic necrosis and gallbladder ischemia had been noted as the major complications after hepatic artery embolization after major liver trauma [16]. There are no literatures thoroughly addressing above etiology. I suspect a potential contributor to this result is impaired perfusion of the bile duct epithelium after TAE. Because of the damage control concept in trauma management, non-selective TAE is often applied in liver trauma. Therefore, the adverse effects of tissue ischemia after TAE are more extensive and profound. For patients who received TAE after blunt liver trauma, the bile duct epithelium sustained dual injuries: the mechanical tissue destruction during trauma and the ischemia injury after TAE. A poor healing process of the injuried bile duct is highly possible and the bile leaks occurred subsequently. Therefore, I suspect TAE is a significant risk factor for major bile duct injury.

\section{Conclusion}

Major bile leak after BLT is uncommon. In addition to a high grade injury, centrally-located liver injuries and initial TAE are also a significant risk factor for major bile duct injury. When patients after BLT are suffering from high fever, persistent abdominal pain or fullness, gross jaundice, we perform abdominal CT to detect intraabdominal fluid collection. If we aspirated bile from intraabdominal fluid, we made a diagnosis of bile duct injury highly possible and ERCP therapy was indicated due to high possibility of major bile leak and to provide early intervention.

\section{Conflicts of Interest}

The author declares no conflicts of interest regarding the publication of this paper.

\section{References}

[1] Shah, J.N. (2007) Endoscopic Treatment of Bile Leaks: Current Standards and Recent Innovation. Gastrointestinal Endoscopy, 6, 1069-1072.

https://doi.org/10.1016/j.gie.2007.02.022 
[2] Singh, V., Narasimhan, K.L., Verma, G.R., et al. (2007) Endoscopic Management of Traumatic Hepatobiliary Injuries. Journal of Gastroenterology and Hepatology, 22, 1205-1209. https://doi.org/10.1111/j.1440-1746.2006.04780.x

[3] Hollands, M.J. and Little, J.M. (1991) Post-Traumatic Bile Fistulae. The Journal of Trauma, 31, 117-120. https://doi.org/10.1097/00005373-199101000-00023

[4] Castagnetti, M., Houben, C., Patel, S., et al. (2006) Minimally Invasive Management of Bile Leaks after Blunt Liver Trauma in Children. Journal of Pediatric Surgery, 41, 1539-1544. https://doi.org/10.1016/j.jpedsurg.2006.05.007

[5] Spinn, M.P. and Adler, D.G. (2006) Successful Endoscopic Therapy of Intrahepatic Bile Leaks Following Hepatic Gunshot Injury: Report of Two Cases. Endoscopy, 38, 859. https://doi.org/10.1055/s-2006-925383

[6] Kapoor, S. and Nundy, S. (2012) Bile Duct Leaks from the Intrahepatic Biliary Tree: A Review of Its Etiology, Incidence and Management. HPB Surgery, 2012, Article ID 752932. https://doi.org/10.1155/2012/752932

[7] Sharma, B.C., Mishra, S.R., Kumar, R., et al. (2009) Endoscopic Management of Bile Leaks after Blunt Abdominal Trauma. Journal of Gastroenterology and Hepatology, 24, 757-761. https://doi.org/10.1111/j.1440-1746.2008.05703.x

[8] Bridges, A., Wilcox, C.M. and Varadarajulu, S. (2007) Endoscopic Management of Traumatic Bile Leaks. Gastrointestinal Endoscopy, 65, 1081-1085. https://doi.org/10.1016/j.gie.2006.11.038

[9] Spinn, M.P., Patel, M.K., Cotton, B.A., et al. (2013) Successful Endoscopic Therapy of Traumatic Bile Leaks. Case Reports in Gastroenterology, 7, 56-62. https://doi.org/10.1159/000346570

[10] Yuan, K.C., Wong, Y.C., Fu, C.Y., et al. (2014) Screening and Management of Major Bile Leak after Blunt Liver Trauma: A Retrospective Single Center Study. Scandinavian Journal of Trauma, Resuscitation and Emergency Medicine, 22, 26. https://doi.org/10.1186/1757-7241-22-26

[11] Lubezky, N., Konikoff, F.M., Rosin, D., et al. (2006) Endoscopic Sphincterotomy and Temporary Internal Stenting for Bile Leaks Following Complex Hepatic Trauma. British Journal of Surgery, 93, 78-81. https://doi.org/10.1002/bjs.5195

[12] Sugiyama, M., Atomi, Y., Matsuoka, T., et al. (2000) Endoscopic Biliary Stenting for Treatment of Persistent Biliary Fistula after Blunt Hepatic Injury. Gastrointestinal Endoscopy, 51, 42-44. https://doi.org/10.1016/S0016-5107(00)70385-3

[13] Bajaj, J.S., Spinelli, K.S. and Dua, K.S. (2006) Postoperative Management of Noniatrogenic Traumatic Bile Duct Injuries: Role of Endoscopic Retrograde Cholangiopancreatography. Surgical Endoscopy, 20, 974-977. https://doi.org/10.1007/s00464-005-0472-3

[14] Wahl, W.L., Brandt, M.M., Hemmila, M.R. and Arbabi, S. (2005) Diagnosis and Management of Bile Leaks after Blunt Injury. Surgery, 138, 742-747. https://doi.org/10.1016/j.surg.2005.07.021

[15] Pachter, H.L., Knudson, M.M., Estrig, B., et al. (1996) Status of Nonoperative Management of Blunt Hepatic Injuries in 1995: A Multicenter Experience with $404 \mathrm{~Pa}$ tients. Journal of Trauma, 40, 31-38. https://doi.org/10.1097/00005373-199601000-00007

[16] Letoublonb, C., Morra, I., Chen, Y., et al. (2011) Hepatic arterial Embolization in the Management of Blunt Hepatic Trauma: Indications and Complications. Journal of Trauma, 70, 1032-1036. https://doi.org/10.1097/TA.0b013e31820e7ca1 